\title{
An Elastoplastic Three Dimensional Homogenization Model for Particle Reinforced Composites
}

\author{
M. Galli ${ }^{\mathrm{a}, \mathrm{b}, *}$ J. Botsis ${ }^{\mathrm{b}}$ J. Janczak-Rusch ${ }^{\mathrm{a}}$ \\ ${ }^{a}$ Laboratory for Surface and Joining Technology, Swiss Federal Laboratories for \\ Materials Testing and Research (EMPA), Dübendorf, 8600, Switzerland \\ ${ }^{\mathrm{b}}$ Laboratoire de mécanique appliquée et d'analyse de fiabilité, Ecole Polytechnique \\ Fédérale de Lausanne (EPFL), Lausanne, 1015, Switzerland
}

\begin{abstract}
A model for the homogenization of the elastoplastic properties of particle reinforced composites is proposed. The microstructure is described by means of a novel technique, consisting of generating particles in a pre-existent constrained Delaunay tetrahedralization of a cubic volume by means of a modified random adsorption algorithm. This technique allows generating models with different amounts of reinforcement by using the same finite element mesh. The obtained particle morphology is similar to that of many ceramic powders often used as reinforcement. Homogenization is carried out for a typical particle reinforced metal matrix composite with reinforcement volume fractions up to 0.25 and the representative volume element size is assessed for both elastic and elastoplastic behaviours. In this latter case the representative volume element size depends on the amount of plastic strain which develops in the matrix material and a criterion to assess the model representativeness is proposed based on the amount of elastic energy stored in the composite. The predictions of the model compare well with pertinent experimental data reported in literature.
\end{abstract}

Key words: Particle reinforced composites, Homogenization method, RVE, Plasticity, Random Sequential Adsorption

* Corresponding author. Tel.: +41216935973 Fax.: +41 216933553

Email address: matteo.galli@epfl.ch (M. Galli).

$U R L:$ http://people.epfl.ch/matteo.galli (M. Galli). 


\section{Introduction}

Particle reinforced or particulate composites have emerged as possible alternatives to conventional fiber reinforced composites. For example, particle reinforced metal matrix composites (PRMMCs) have shown great potential and have been the object of intense research for the last twenty years, especially in the aerospace field: typically, these materials consist of an aluminium alloy matrix reinforced with ceramic particles. Another important family of particulate composites is that of reinforced polymers with many applications for instance in the automotive industry. The particulate morphology can also be found in a number of other material systems: reinforced concrete can also be thought of as a particle reinforced composite or ceramics can be modelled as particulate composites and are candidate materials for many high temperature applications. Consequently, the characterization and modelling of the mechanical behaviour of such materials are fundamental to their reliable use. The existing modelling approaches can be divided into two main groups [1]: the first includes methods which statistically describe the microstructures (mean field approaches and variational bounding methods) while the second those which are based on modelling discrete microstructures (periodic microfield, embedded cell and windowing approaches). Thanks to the progressive increase of available computing power, several models of the latter type have been proposed in recent years to study both linear and non-linear mechanical behaviours of heterogeneous systems. However, most reported models are two dimensional. The morphology of the microstructure can be either directly derived from an experimental image, on which a finite element mesh is superposed, e.g. [2, 3], or reconstructed by assigning the particles a shape, usually circular or rectangular, e.g. [4]. Although they capture some of the physical aspects of the actual material properties, planar models are not able to predict the effective behaviour of composites with randomly distributed particles, especially in the elastoplastic range as suggested in [5] and extensively demonstrated in [6]. Plane stress models tend to underestimate the strengthening effect of the particles whereas plane strain models tend to overestimate it. As a consequence, three dimensional models are required.

The geometrical description of a three dimensional microstructure is much more challenging than in the planar case. Experimental techniques to obtain three dimensional images of actual microstructures are complex and expensive and can be used only in rare cases (e.g. in [7, 8] a real three dimensional image of microstructure obtained by holotomography is directly used as input for the model, while in $[9,10]$ the three dimensional microstructure is reconstructed from two dimensional images by means of a serial sectioning process). As for the two dimensional approaches the alternative is constituted by methods in which particles are assumed to have arbitrary shapes (in most cases spherical). These approaches give more accurate results [11], but present two main drawbacks: particles with an imposed shape are not always representative of the 
real microstructure (as in the two dimensional case), moreover the generation and meshing of the geometry can be quite demanding especially if composites with different compositions and particle distributions are to be studied.

In the present work a novel technique to generate the microstructure geometry is proposed. This method allows reproducing the actual shape of ceramic particles normally used in particle reinforced composites. Unlike other methods, particles do not need to be drawn and then meshed but are generated in an existing mesh; this makes it possible to easily model particle reinforced composites with different reinforcement volume fractions since the geometry does not need to be redefined for each composition.

The size of the representative volume element (RVE) is assessed for the thermoelastic behaviour and a procedure is developed to check the representativeness when plasticity is taken into account. In this paper no attempt is made to compare the present results with existing numerical and analytical models, however the developed model is tested on a real PRMMC for which the elastoplastic behaviour both of the matrix metal and of the actual composite are known.

\section{Generation of the multi-particle cell}

A cubic multi-inclusion unit cell microstructural model is proposed. The domain is discretized by the Constrained Delaunay Tetrahedralization (CDT), a variation of the Delaunay tetrahedralization.

The Delaunay tetrahedralization can be defined as follows: Let $S$ be a finite set of points in $\mathbb{R}^{3}$. Four non-coplanar points $s_{i}, s_{j}, s_{k}$ and $s_{l}$ are the vertices of a Delaunay tetrahedron $t$ if and only if there exists a location $x$ which is equally close to $s_{i}, s_{j}, s_{k}$ and $s_{l}$ and closer to $s_{i}, s_{j}, s_{k}$ and $s_{l}$ than to any other $s_{m} \in S$. The location $x$ is the center of circumsphere of $t$.

The decomposition of a three dimensional geometric object $\Omega$ into a tetrahedral mesh implies that the boundary (including internal boundaries and holes) of the object $\partial \Omega$ has to be respected by mesh faces. The Delaunay tetrahedralization of the object vertices generally does not statisfy this requirement. The CDT is a variation of the Delaunay tetrahedralization which can respect an imposed boundary, thus allows meshing domains of a given shape. To define a constrained Delaunay tetrahedron it is necessary to introduce the concept of visibility between two points. The visibility between two points $s_{i}$ and $s_{j}$ is occluded if there is a constraining polygon $f \in \partial \Omega$ such that $s_{i}$ and $s_{j}$ lie on opposite sides of the plane which includes $f$ and the line segment $\overline{s_{i} s_{j}}$ intersects $f$. Four non-coplanar points are the vertices of a constrained Delaunay tetrahedron $t_{c}$ if its circumsphere encloses no $s_{m} \in S$ visible from any location in the relative interior of $t_{c}$. Note that the two definitions are the same except for the fact that in the CDT the portion of volume of the circumsphere which 
lies outside $\partial \Omega$ is ignored.

In our application the CDT is carried out by the software TetGen [12] which ensures quality mesh generation by controlling element distortion (the adopted metric is the maximum radius-edge ratio $r_{\max }$, which is the ratio between the radius of a tetrahedron circumsphere and its shortest edge). Moreover, it provides additional features such as the possibility to limit the maximum tetrahedron volume $v_{\max }^{t}$ and to impose the coordinates of an additional set of vertices $\widetilde{S}$. The latter feature is exploited to create vertices in random positions both on the surface and inside the domain, in order to allow a certain degree of perturbation in the CDT, which otherwise would lead to too regular meshes, not suitable for the purposes of this work.

In the present work the process of generating the multi-inclusion unit cell is divided into two main stages: the discretization of the cell volume and the creation of the microstructure. The first stage is made of four main steps:

(1) Definition of the cell domain $\Omega$ and of its boundary $\partial \Omega$

(2) Creation of a set $\widetilde{S}$ of imposed vertices: $\widetilde{S}=\left\{\widetilde{s}_{1}, \ldots, \widetilde{s}_{n}: \widetilde{s}_{i} \in(\Omega \cup \partial \Omega)\right\}$

(3) Definition of $r_{\max }$ and $v_{\max }^{t}$

(4) CDT of $\Omega$ with respect to $r_{\max }$ and $v_{\max }^{t}$

The composite microstructure is produced in the second stage: the reinforcement volume fraction $V_{r}$, the minimum and maximum particle volume $\left(v_{\min }^{p}\right.$ and $v_{\max }^{p}$, respectively) are imposed and particles are generated in the tetrahedralized cubic domain according to the following iterative procedure:

(1) Choice of a random vertex $s$ as nucleation point for a particle $p$ : the particle is the set of tetrahedra which share $s: p=\left\{t_{1}, \ldots, t_{n}: s \in t_{i}\right\}$

(2) Test on the particle volume: if the particle volume does not satisfy the prescribed constraints the particle is discarded and another random vertex is chosen

(3) Tests on the particle position: the particle is discarded if it overlaps (shares a tetrahedron $t_{k}$ ) or touches (shares a facet $f_{k}$ ) another particle, nevertheless particles are allowed to share edges

(4) The particle $p$ is included in the set $P$ of valid particles:

$$
P=\left\{p_{1}, \ldots, p_{n}: v_{\text {min }}^{p} \leq v_{i}^{p} \leq v_{\text {max }}^{p} ; \forall f_{k}, t_{k} \in p_{i}, \forall p_{j} \in P \Rightarrow f_{k}, t_{k} \notin p_{j}\right\}
$$

The procedure is repeated until the design reinforcement volume fraction $V_{r}$ is obtained (the algorithm flowchart is reported in figure 1). The proposed approach is suitable to obtain statistically homogeneous particle distributions and is a variant of the Random Sequential Adsorption (RSA), in which particles placed sequentially at random positions are accepted if they do not overlap any of the formerly created particles and rejected otherwise [13]. The RSA has attracted a lot of attention in the last thirty years and has been applied in several fields, e.g. to reconstruct dispersions [14]. The most immediate way to characterize the RSA is by means of the jamming limit, which is the 


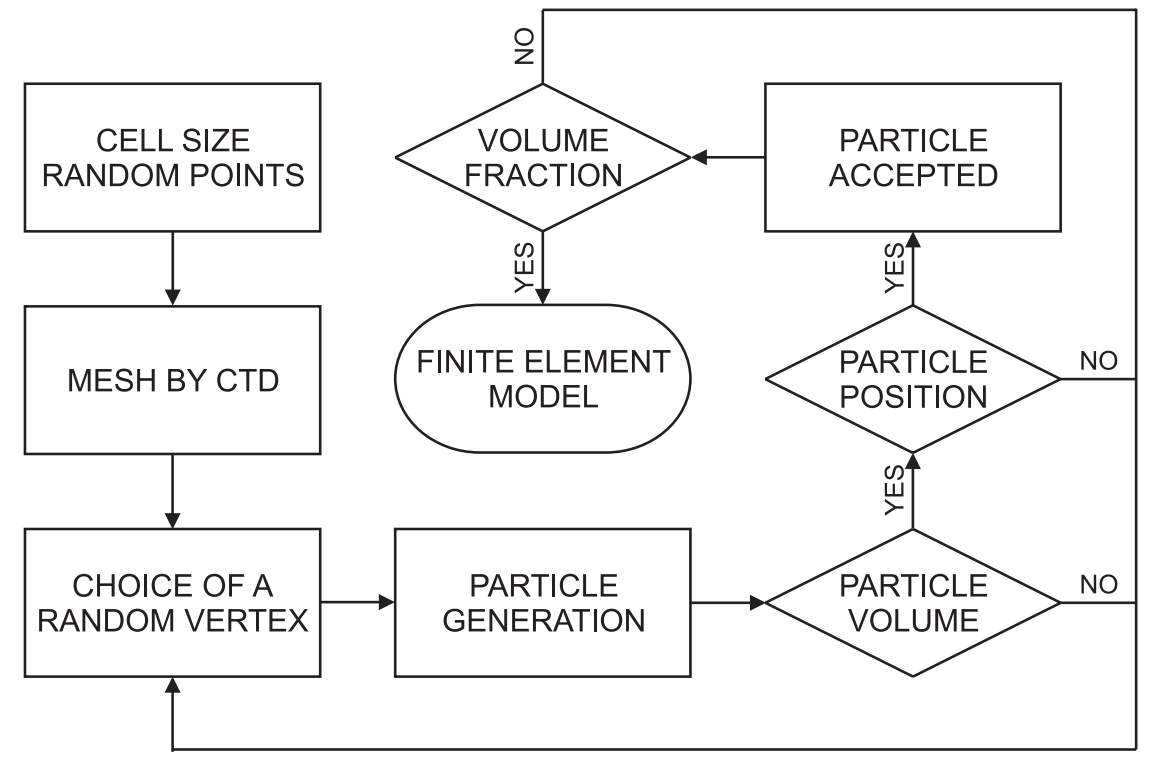

Fig. 1. Flowchart of the algorithm developed to generate the model.

maximum obtainable inclusion volume fraction and has been determined for different particle shapes, e.g. for two dimensional discs it is $0.547 \pm 0.002$ while for aligned squares it is $0.562 \pm 0.002$ [15].

The present algorithm has been applied to study almost monodisperse reinforcement powders (a 13\% tolerance on the particle volume is allowed) randomly distributed in the volume under consideration (figure 2). Typically one particle consists of about 24-26 elements (12-13 in case of particles generated from nodes lying on the cube faces) and exhibits an almost convex shape which resembles that of ceramic powders actually used in composites, which are made of irregular polyhedral particles (figure 3).

To obtain a rough estimate of the jamming limit, tests were carried out with up to $10^{7}$ iterations and a value of about 0.26 was observed (in the case of identical spheres it is $0.382 \pm 0.003$ [16]). Note that particles were allowed to share edges in order to raise the jamming limit in the case of monodisperse reinforcement. However, in the case of a composite with 0.25 reinforcement volume fraction (the largest considered in the present work), the number of shared edges is less than $4 \%$ of the total number of edges on the surface of particles (the average particle surface consists of 36-39 edges). Moreover, due to the way particles are generated, one edge can be shared by no more than two particles and shared edges are randomly oriented. Thus it is very unlikely to find in the model chains of shared edges sufficiently long to significantly influence the strain field in the matrix material. Following these considerations it is assumed that the effect of particle shared edges on the model global response is negligible.

To achieve higher volume fractions without relaxing the constraints on the particle size distribution, more sophisticated algorithms are required: one possible strategy could be to identify all the particles having a compatible size 


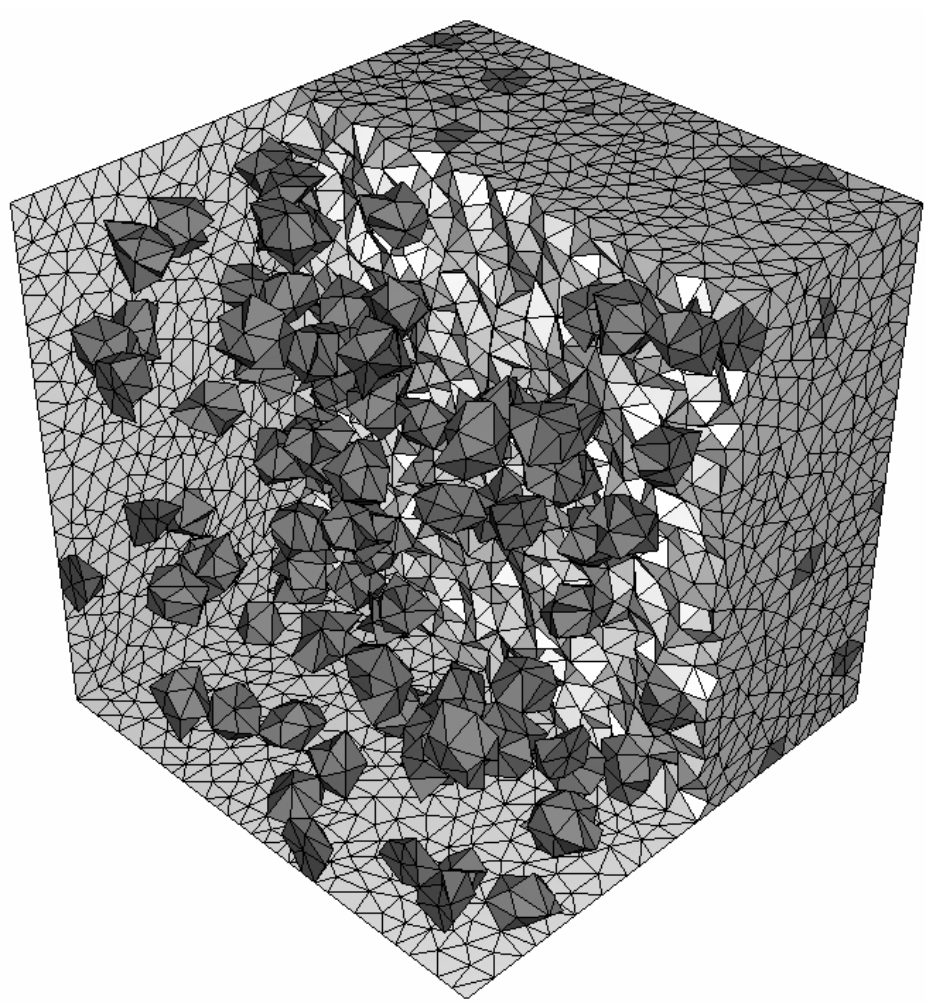

Fig. 2. An example of a unit cell with $V_{r}=0.05$ (part of the matrix elements are not displayed to show the particles).
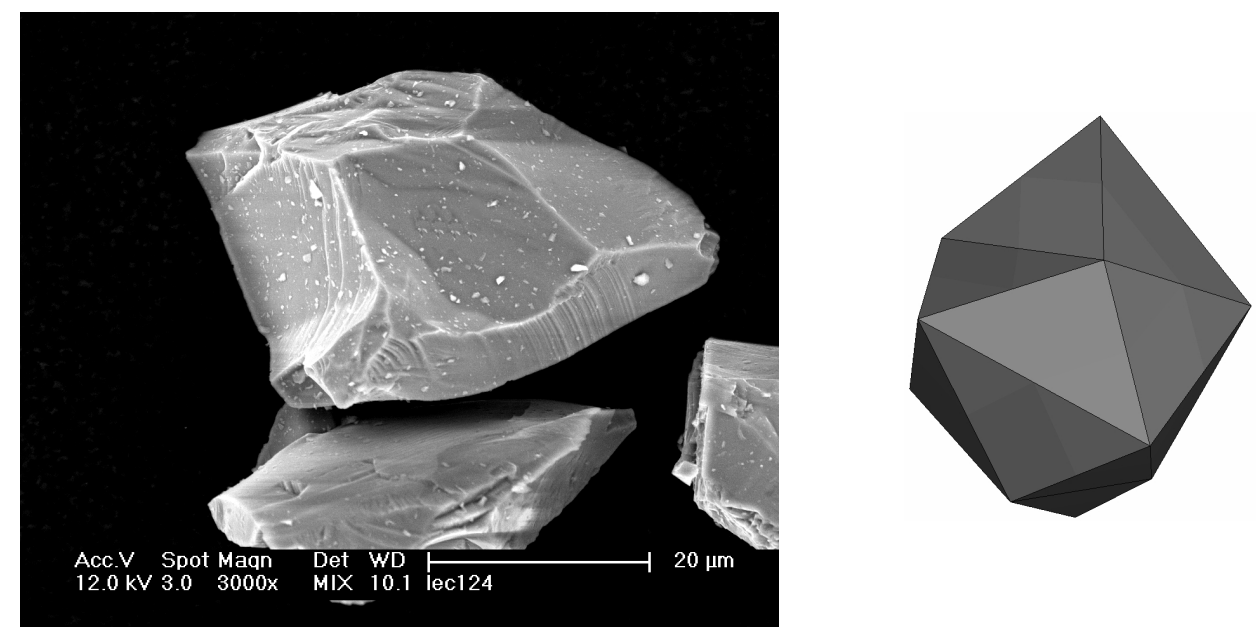

Fig. 3. A typical $\mathrm{SiC}$ powder used as reinforcement in composites (image obtained by scanning electron microscopy) and a typical model particle.

and then to choose those leading to a higher $V_{r}$.

The obtained finite element model is solved and post processed with ABAQUS ${ }^{\circledR}$ software package. Quadratic elements are employed to model the thermoelastic behaviour while in elastoplasticity modified 10-node elements are employed to avoid possible volumetric locking in yielded matrix regions. Perfect adhesion between particles and matrix is assumed throughout the whole study. 


\section{Elastic behaviour}

For heterogeneous elastic materials admitting a RVE, the effective stiffness tensor $\bar{C}_{i j k l}$ and the effective compliance tensor $\bar{D}_{i j k l}$ are linked to the average stress tensor $\langle\sigma\rangle_{i j}$ and the average strain tensor $\langle\epsilon\rangle_{i j}$

$$
\begin{gathered}
\langle\sigma\rangle_{i j}=\bar{C}_{i j k l}\langle\epsilon\rangle_{k l} \\
\langle\epsilon\rangle_{i j}=\bar{D}_{i j k l}\langle\sigma\rangle_{k l}
\end{gathered}
$$

The macroscopic responses $\langle\sigma\rangle_{i j}$ and $\langle\epsilon\rangle_{i j}$ are defined as

$$
\begin{aligned}
\langle\sigma\rangle_{i j} & =\frac{1}{\Omega} \int_{\Omega} \sigma_{i j}(\mathbf{x}) d \Omega \\
\langle\epsilon\rangle_{i j} & =\frac{1}{\Omega} \int_{\Omega} \epsilon_{i j}(\mathbf{x}) d \Omega
\end{aligned}
$$

where $\Omega$ stands for the volume of the region under consideration while $\sigma_{i j}(\mathbf{x})$ and $\epsilon_{i j}(\mathbf{x})$ are the local stress and strain fields.

Comparison of (1) with (2) yields

$$
\bar{C}_{i j k l}=\left(\bar{D}_{i j k l}\right)^{-1}
$$

By definition the effective stiffness tensor $\bar{C}_{i j k l}$ and the effective compliance tensor $\bar{D}_{i j k l}$ are assumed to be boundary condition independent. Actually $\Omega$ can be considered a RVE when the responses under static and kinematic uniform boundary conditions coincide [17]. This approach in principle is valid only for bodies of infinite dimensions, nevertheless, if applied on sufficiently large volumes, leads to very precise estimates of the effective mechanical properties.

To evaluate the stiffness tensor under kinematic uniform boundary conditions $\bar{C}_{i j k l}^{\langle\epsilon\rangle}$ six simulations are required, one for each column (the matrix notation is employed):

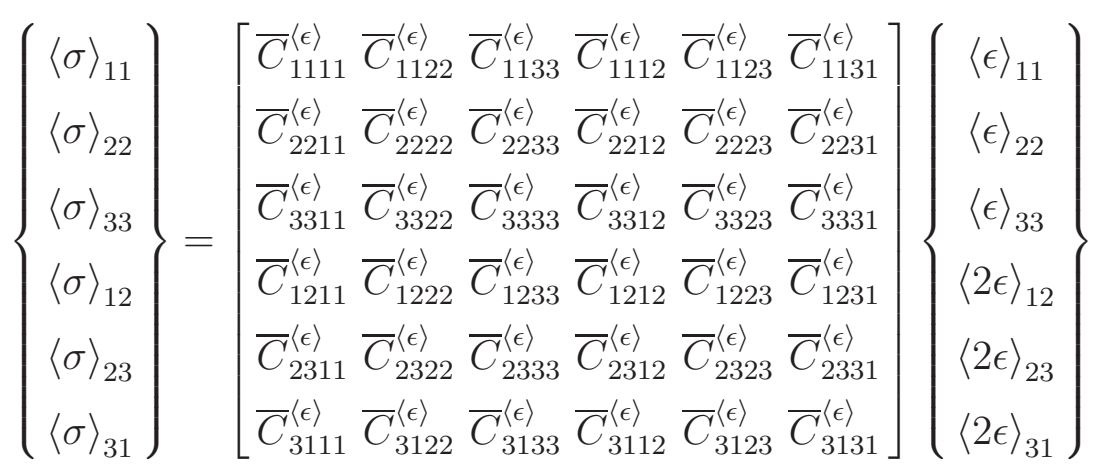

With the same procedure the compliance tensor under static uniform boundary conditions $\bar{D}_{i j k l}^{\langle\sigma\rangle}$ can be calculated along with its inverse $\bar{C}_{i j k l}^{\langle\sigma\rangle}$, the stiffness 
Table 1

\begin{tabular}{cccc}
\hline Material & $E[\mathrm{GPa}]$ & $\nu$ & $\mathrm{CTE}\left[{ }^{\circ} \mathrm{C}^{-1}\right]$ \\
\hline Matrix & 73 & 0.33 & $2.15 \cdot 10^{-5}$ \\
Particles & 480 & 0.17 & $3.8 \cdot 10^{-6}$ \\
\hline
\end{tabular}

Young's moduli, Poisson's ratios and coefficients of thermal expansion of the components.

Table 2

\begin{tabular}{ccc}
\hline N. Particles & Vol. Frac. & $\xi$ \\
\hline 6 & 0.257 & 2.85 \\
46 & 0.252 & 5.69 \\
150 & 0.251 & 8.43 \\
360 & 0.250 & 11.29 \\
705 & 0.250 & 14.12 \\
\hline
\end{tabular}

Unit cell models for the assessment of the RVE size.

tensor under static uniform boundary conditions

$$
\bar{C}_{i j k l}^{\langle\sigma\rangle}=\left(\bar{D}_{i j k l}^{\langle\sigma\rangle}\right)^{-1}
$$

The RVE size is achieved when the difference $\left(\bar{C}_{i j k l}^{\langle\epsilon\rangle}-\bar{C}_{i j k l}^{\langle\sigma\rangle}\right)$ is negligible, typically less than $5 \%$.

Homogenization was carried out for the elastic behaviour of a typical particle reinforced metal matrix composite with the mechanical properties of the components shown in table 1 [18]. In this study composites with amounts of reinforcement up to $V_{r}=0.25$ were considered. The RVE size was assessed for the composite with the largest amount of reinforcement and five unit cells of increasing dimensions were modelled: the smallest contained 6 particles while the largest 705. The main features of the models are summarized in table 2 . To measure the cell size with respect to the particle dimension the metric $\xi$ is defined:

$$
\xi=\sqrt[3]{\frac{V_{u c}}{\bar{V}_{p}}}
$$

where $V_{u c}$ is the unit cell volume and $\bar{V}_{p}$ is the average particle volume.

It can be seen that the developed algorithm allows to obtain the prescribed composition very precisely: even in the case of the smallest volume the difference between the prescribed and the actual reinforcement volume fraction is less than $1 \%$ of the total volume. Effective stiffness tensors under static and kinematic uniform boundary conditions were determined for all the models and average stress and strain components (3) and (4) were computed by weighting the values in each integration point by the associated volume. 


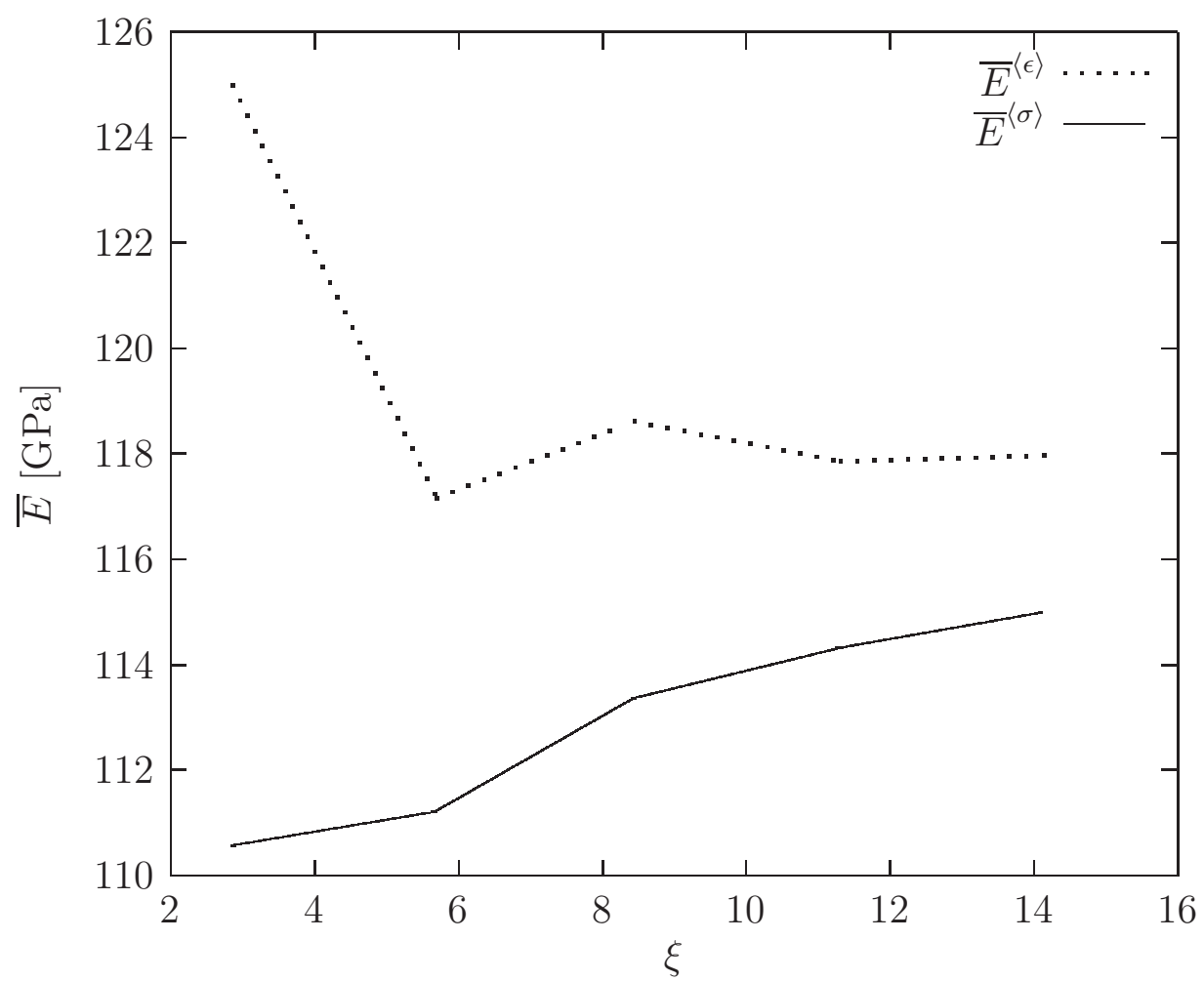

Fig. 4. Young's modulus $\bar{E}$ under kinematic and static uniform boundary conditions as a function of the unit cell size.

In figure 4 the calculated Young's moduli are reported. The difference between the Young's moduli calculated under kinematic and static uniform boundary conditions $\bar{E}^{\langle\epsilon\rangle}$ and $\bar{E}^{\langle\sigma\rangle}$ is already about $3 \%$ for the 360 particle model, however for the sake of consistency with the rest of the work the composite effective elastic properties were computed for the cell with the largest size $(\xi=14.12)$. The calculated stiffness tensor was that of a linear elastic isotropic material. No appreciable difference could be observed between the shear modulus as a function of Young's Modulus and Poisson's ratio and that obtained in case of pure shear boundary conditions. The results for all the considered compositions are reported in table 3.

Note that all the models provide results which respect the Hashin-Shtrikman bounds [19] as shown by the data obtained for the shear modulus in figure 5 .

\section{Thermoelastic behaviour}

If thermal expansion is taken into account and a linear thermoelastic behaviour is assumed for both the matrix and the reinforcement, the overall stress-strain relations can be written in the form

$$
\langle\epsilon\rangle_{i j}=\bar{D}_{i j k l}\langle\sigma\rangle_{k l}+\bar{\alpha}_{i j}^{\langle\sigma\rangle} \Delta T
$$


Table 3

\begin{tabular}{ccccc}
\hline$V_{r}$ & $\begin{array}{c}E \\
{[\mathrm{MPa}]}\end{array}$ & $\nu$ & $\begin{array}{c}G(E, \nu) \\
{[\mathrm{MPa}]}\end{array}$ & $\begin{array}{c}G \\
{[\mathrm{MPa}]}\end{array}$ \\
\hline 0.05 & 79973 & 0.323 & 30226 & 30433 \\
0.10 & 87897 & 0.315 & 33414 & 33714 \\
0.15 & 96538 & 0.308 & 36912 & 37427 \\
0.20 & 106784 & 0.299 & 41107 & 41661 \\
0.25 & 117984 & 0.290 & 45725 & 46397 \\
\hline
\end{tabular}

Elastic properties of the different composites.

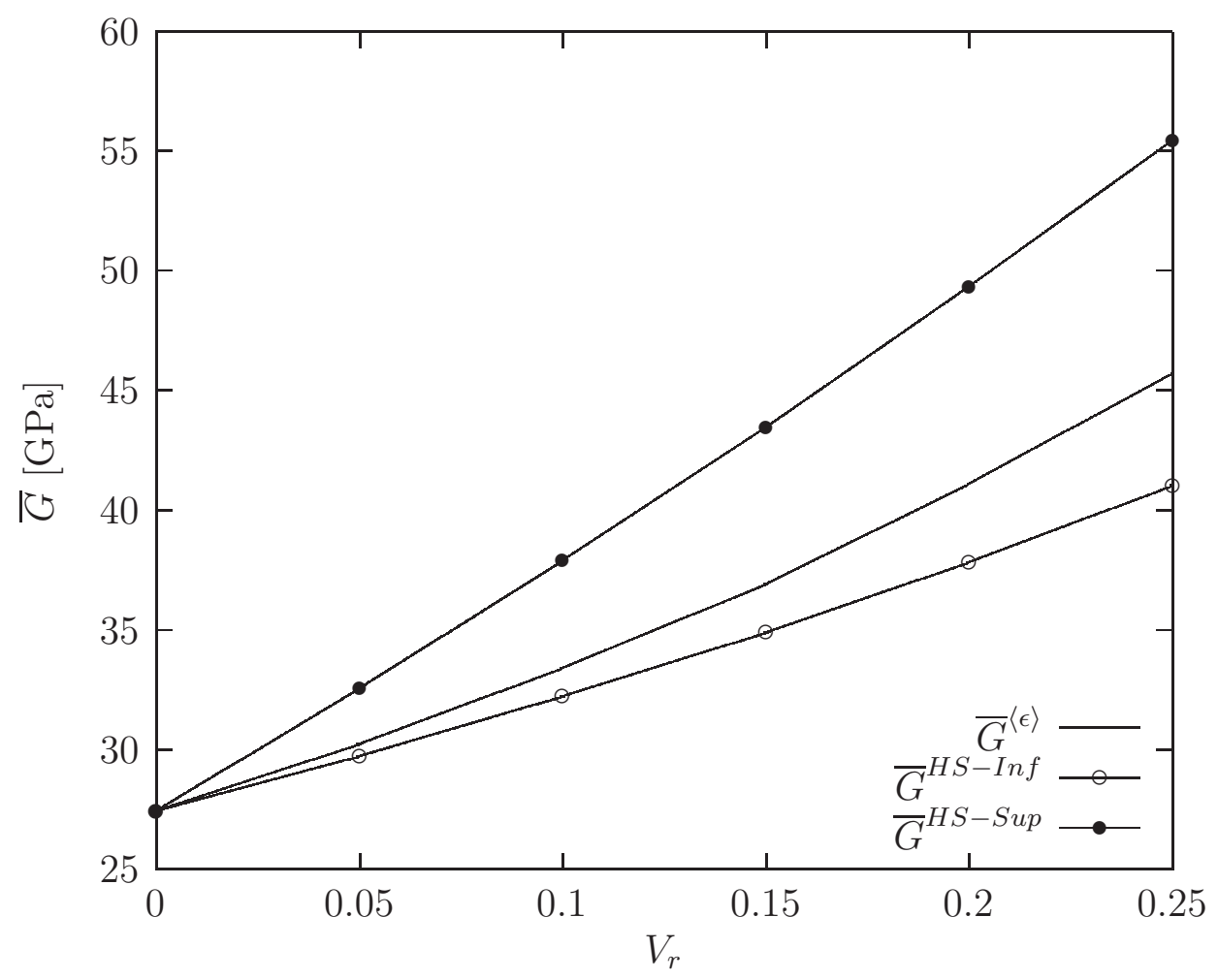

Fig. 5. Comparison between the calculated shear moduli and Hashin-Shtrikman bounds.

$$
\langle\sigma\rangle_{i j}=\bar{C}_{i j k l}\left(\langle\epsilon\rangle_{k l}-\bar{\alpha}_{i j}^{\langle\epsilon\rangle} \Delta T\right)
$$

where $\bar{\alpha}_{i j}^{\langle\sigma\rangle}$ and $\bar{\alpha}_{i j}^{\langle\epsilon\rangle}$ are the effective thermal expansion tensors under kinematic and static uniform boundary conditions, respectively; $\Delta T$ is a spatially uniform temperature difference with respect to the stress-free reference temperature. Comparison of (9) and (10) with (5) yields to

$$
\bar{\alpha}_{i j}^{\langle\sigma\rangle}=\bar{\alpha}_{i j}^{\langle\epsilon\rangle}
$$




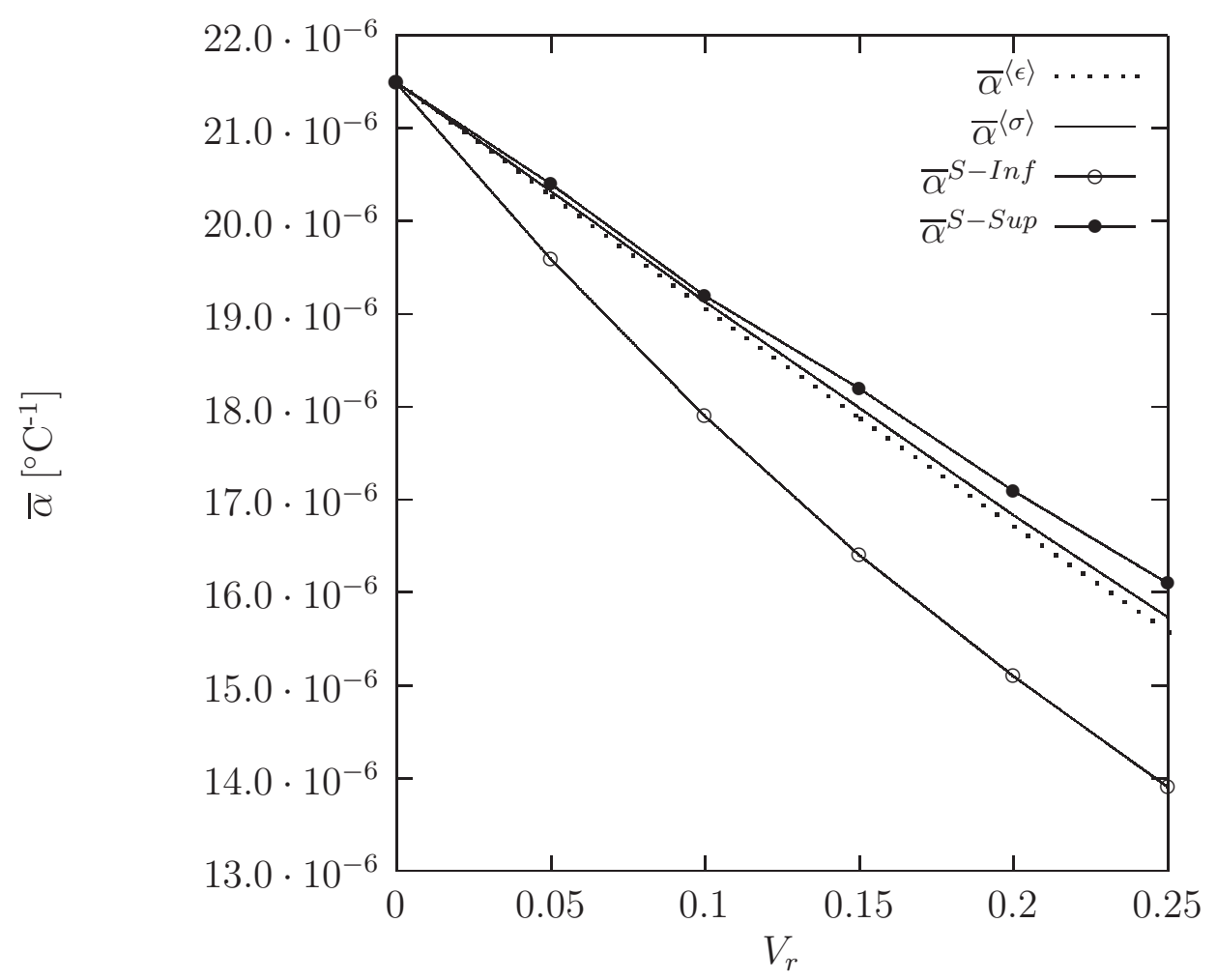

Fig. 6. Comparison between Schapery's bounds and the calculated coefficients of thermal expansions $\bar{\alpha}$ under static and kinematic uniform boundary conditions.

There is no need to check the size of the RVE for the CTE since $\bar{\alpha}_{i j}$ for twophase materials is directly related to the elastic moduli of the composite [20]. Simulations were carried out under both types of boundary conditions on the same unit cells adopted to characterize the elastic behaviour and the material proved to be isotropic

$$
\bar{\alpha}_{i j} \approx \delta_{i j} \bar{\alpha}
$$

where $\delta_{i j}$ is Kronecker delta. The calculated CTEs for the different compositions and the comparison with the Schapery's bounds [21] are depicted in figure 6 .

\section{Elastoplastic behaviour}

In the case of linear elastic statistically isotropic particle reinforced composites, cubic cells with edges five times the inclusion characteristic size lead to very precise effective moduli as indicated in [22] and confirmed in the present work: the difference between the computed effective Young's moduli under static and kinematic uniform boundary conditions is about $5 \%$ for the cell with $\xi=5.69$. For elastoplastic matrix behaviours, considerably larger cells are required to achieve the RVE size, especially when large plastic strain develops, because 
the strain field is significantly more inhomogeneous than in the elastic case. In such cases a criterion to define the RVE is needed.

The same typical particle reinforced metal matrix composite considered for the elastic behaviour was modelled. The matrix was assumed to be elastoplastic and to follow the Von Mises yield criterion

$$
\varphi\left(\sigma_{i j}, \epsilon^{p}\right)=\sqrt{3 J_{2}^{\prime}}-k\left(\epsilon^{p}\right)
$$

where $\epsilon^{p}$ is the equivalent plastic strain, $J_{2}^{\prime}$ is the second invariant of the stress deviator and $k\left(\epsilon^{p}\right)$ is the isotropic hardening law with the form

$$
k\left(\epsilon^{p}\right)=\sigma_{y}+h\left(\epsilon^{p}\right)^{q}
$$

where $\sigma_{y}, h$ and $q$ are the yield stress and the strain hardening parameters, respectively. The matrix material is assumed to have the elastic properties reported in table 1 , the uniaxial yield stress $\sigma_{y}=170 \mathrm{MPa}$, the strain hardening paremeters $h=577 \mathrm{MPa}$ and $q=0.37$ [18]. Preliminary studies showed that, for up to 0.05 imposed strain, the model response did not vary significantly whether small or large displacements were considered. Thus the simulations were carried out within the framework of the small displacements assumption. Due to the presence of particles the stress-strain curve of the composite is expected to present a larger hardening than that of the matrix material. This effect can be described by following the approach proposed in [23], in which the microstress $\sigma_{i j}(\mathbf{x})$ is split into two parts: the one which would occur if the constituents were elastic and a self equilibrated residual stress field

$$
\sigma_{i j}(\mathbf{x})=L_{i j k l}(\mathbf{x})\langle\sigma\rangle_{k l}+\sigma_{i j}^{r}(\mathbf{x})
$$

where $L_{i j k l}(\mathbf{x})$ stands for the elastic stress localization tensor. The average elastic energy in the material can be written as

$$
\langle U\rangle=\frac{1}{2 \Omega} \int_{\Omega} D_{i j k l}(\mathbf{x}) \sigma_{i j}(\mathbf{x}) \sigma_{k l}(\mathbf{x}) d \Omega
$$

where $D_{i j k l}(\mathbf{x})$ is the local compliance tensor. By introducing (15) in (16) the following expression is obtained

$$
\langle U\rangle=\frac{1}{2} \bar{D}_{i j k l}\langle\sigma\rangle_{i j}\langle\sigma\rangle_{k l}+\frac{1}{2 \Omega} \int_{\Omega} D_{i j k l}(\mathbf{x}) \sigma_{i j}^{r}(\mathbf{x}) \sigma_{k l}^{r}(\mathbf{x}) d \Omega
$$

The first term is the average macroscopic elastic energy $\bar{U}$ while the second is the average micro-stored elastic energy due to the residual stresses $\left\langle U^{r}\right\rangle$. For the sake of simplicity (17) is rewritten as

$$
\langle U\rangle=\bar{U}+\left\langle U^{r}\right\rangle
$$


In an analogous manner the average dissipated energy $\langle P\rangle$ can be computed

$$
\langle P\rangle=\int_{0}^{t} \frac{1}{\Omega} \int_{\Omega} \sigma_{i j}(\mathbf{x}) \dot{\epsilon}_{i j}^{p}(\mathbf{x}) d \Omega d t
$$

where $\dot{\epsilon}_{i j}^{p}(\mathbf{x})$ is the local plastic strain rate. At the macroscopic level the plastic strain is the difference between the average total strain and the macroscopic elastic strain

$$
\bar{\epsilon}_{i j}^{p}=\langle\epsilon\rangle_{i j}-\bar{D}_{i j k l}\langle\sigma\rangle_{i j}
$$

note that

$$
\bar{\epsilon}_{i j}^{p} \neq \frac{1}{\Omega} \int_{\Omega} \epsilon_{i j}^{p}(\mathbf{x}) d \Omega
$$

By introducing (15) in (19) the following expression for the average macroscopic plastic work is obtained

$$
\int_{0}^{t}\langle\sigma\rangle_{i j} \dot{\bar{\epsilon}}_{i j}^{p} d t=\langle P\rangle+\left\langle U^{r}\right\rangle
$$

Therefore two contributions to the average plastic work at the macroscopic level are identified: the average dissipated energy $\langle P\rangle$ and the average elastic energy due to residual stresses $\left\langle U^{r}\right\rangle$, which is stored in the material and contributes to the macroscopic hardening (for further details refer to [23]).

In the present work the uniaxial tensile behaviour of the same increasingly larger cells (except for that with $\xi=2.85$ ) modelled in the framework of linear elasticity was considered (table 2). Their response was studied under four different sets of boundary conditions, all of them representative of a pure tensile test (additional boundary conditions to constrain rigid body displacements 
are not indicated):

$$
\begin{aligned}
& \left\{\begin{array}{l}
u_{1}\left(0, x_{2}, x_{3}\right)=0 \\
u_{1}\left(L, x_{2}, x_{3}\right)=\bar{u} \\
u_{2}\left(x_{1}, 0, x_{3}\right)=0 \\
\left.u_{2}\left(x_{1}, L, x_{3}\right)\right|_{x_{2}, x_{3} \neq L}=u_{2}(0, L, L) \\
u_{3}\left(x_{1}, x_{2}, 0\right)=0 \\
\left.u_{3}\left(x_{1}, x_{2}, L\right)\right|_{x_{2}, x_{3} \neq L}=u_{3}(0, L, L)
\end{array}\right. \\
& \left\{\begin{array}{l}
f_{1}\left(0, x_{2}, x_{3}\right)=-\bar{p} \\
f_{1}\left(L, x_{2}, x_{3}\right)=\bar{p} \\
u_{2}\left(x_{1}, 0, x_{3}\right)=0 \\
\left.u_{2}\left(x_{1}, L, x_{3}\right)\right|_{x_{2}, x_{3} \neq L}=u_{2}(0, L, L) \\
u_{3}\left(x_{1}, x_{2}, 0\right)=0 \\
\left.u_{3}\left(x_{1}, x_{2}, L\right)\right|_{x_{2}, x_{3} \neq L}=u_{3}(0, L, L)
\end{array}\right. \\
& \begin{cases}u_{1}\left(0, x_{2}, x_{3}\right)=0 \\
u_{1}\left(L, x_{2}, x_{3}\right)=\bar{u} \\
f_{2}\left(x_{1}, 0, x_{3}\right)=0 \\
f_{2}\left(x_{1}, L, x_{3}\right)=0 \\
f_{3}\left(x_{1}, x_{2}, 0\right)=0 \\
f_{3}\left(x_{1}, x_{2}, L\right)=0\end{cases} \\
& \begin{cases}f_{1}\left(0, x_{2}, x_{3}\right)=-\bar{p} \\
f_{1}\left(L, x_{2}, x_{3}\right)=\bar{p} \\
f_{2}\left(x_{1}, 0, x_{3}\right)=0 \\
f_{2}\left(x_{1}, L, x_{3}\right)=0 \\
f_{3}\left(x_{1}, x_{2}, 0\right)=0 \\
f_{3}\left(x_{1}, x_{2}, L\right)=0\end{cases}
\end{aligned}
$$

Here $u_{i}$ and $f_{i}$ are the nodal displacement and the surface load in the $x_{i}$ direction and $L$ is the length of the edge of the cell. In (23) all faces are forced to stay planar during the deformation process while in (24) only those parallel to the tensile axis. Boundary conditions (25) are mixed static-kinematic with imposed uniform displacements on the tensile faces while (26) are purely static.

In this work periodic boundary conditions $[6,11,24]$ were not adopted since they require mesh periodicity [25]. However, the effective composite behaviour obtained under mixed static-kinematic boundary conditions $(23,24,25)$ is always bounded by those obtained under static and kinematic uniform boundary conditions $[26]$.

The obtained effective uniaxial stress-strain curves for the unit cell with $\xi=$ 14.12 and $V_{r}=0.25$ are shown in figure 7 . The response of the model depends 


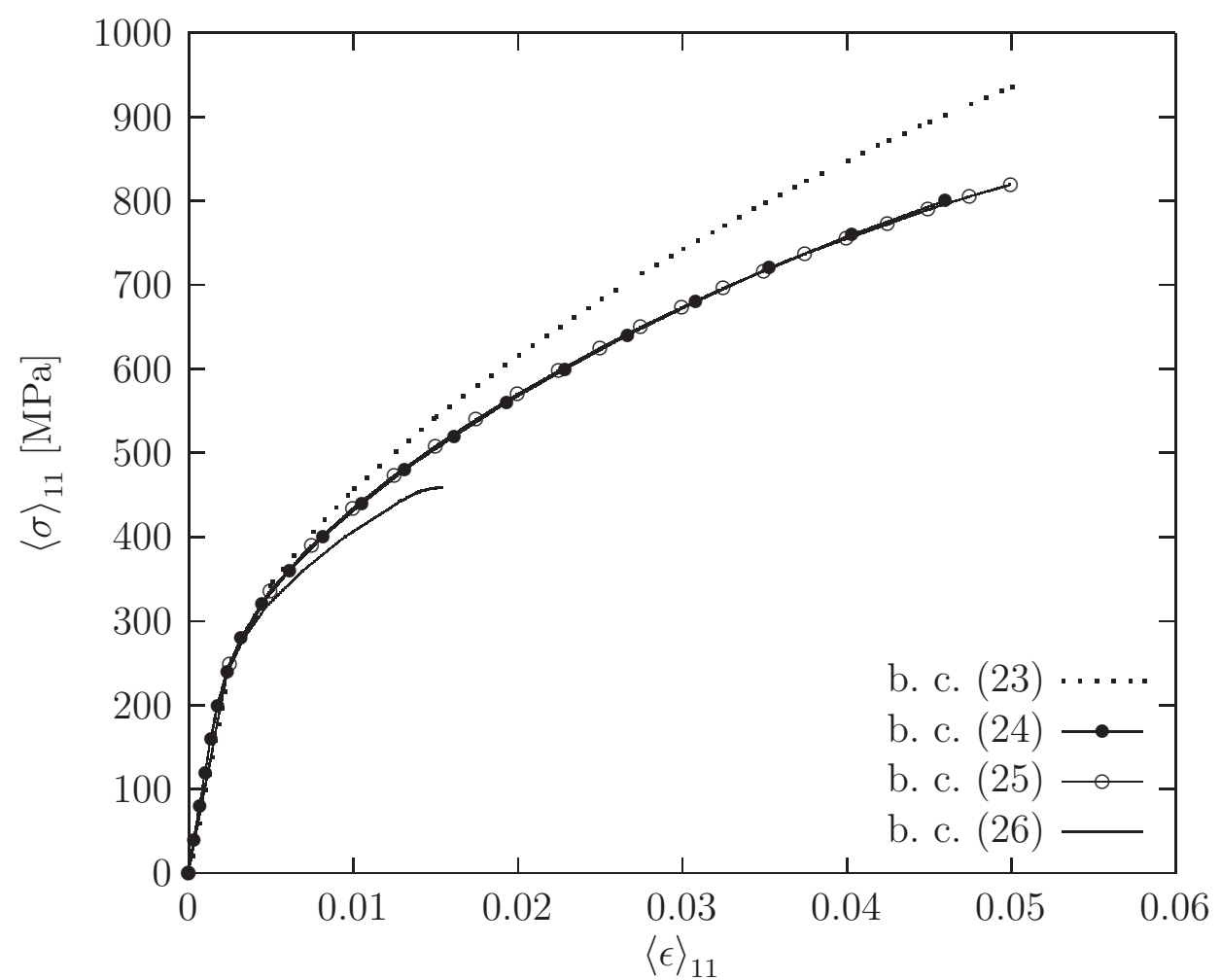

Fig. 7. Effective uniaxial stress-strain curves under different boundary conditions.

on the applied boundary conditions while, if the material were homogeneous, the four different sets of boundary conditions would lead to the same stressstrain curve. Results show that the effective curves obtained by imposing that two or four faces keep planar, (25) and (24), are bounded by those resulting from (23) and (26), where all faces keep planar or are free to deform, respectively. It can also be observed that the curve due to (26) flattens for $\langle\epsilon\rangle_{11}=0.015$. This is caused by the concentration of plastic strain in the corner regions of the cell because of the lack of confinement. This phenomenon increasingly affects the results as the prescribed tension $\bar{p}$ nears the load carrying capacity of the material and eventually leads the model to lose representativeness.

The effective mechanical behaviour of the composite resulting from the model and especially the macroscopic hardening strongly depend on the size of the unit cell (figure 8). To assess the size of the RVE the evolution of $\left\langle U^{r}\right\rangle$ as a function of the cell size $\xi$ was studied for unit cells with $V_{r}=0.25$ : pure traction simulations were carried out under boundary conditions (24) and (25), with applied macrostress $\langle\sigma\rangle_{11}=800 \mathrm{MPa}$ and applied macrostrain $\langle\epsilon\rangle_{11}=0.05$, respectively. The value of $\left\langle U^{r}\right\rangle$ was obtained by means of (18), where $\langle U\rangle$ was computed during the elastoplastic simulation and $\bar{U}$ was evaluated by simulating the material elastic behaviour under (24) and (25). To have a better accuracy, the energy $\bar{U}$ was calculated directly and not by using the results of the homogenization of the elastic properties reported in table 3 . 


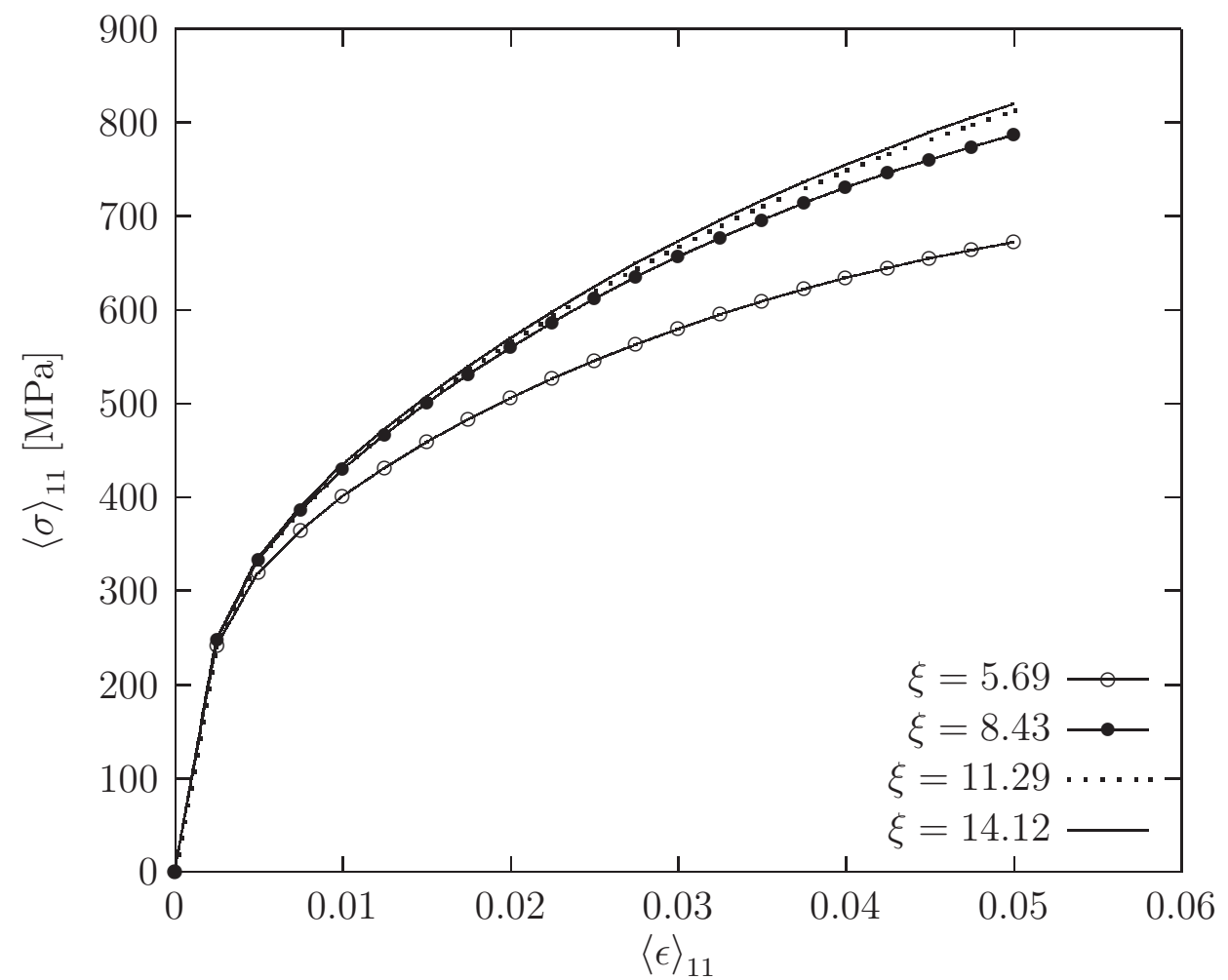

Fig. 8. Effective uniaxial stress-strain curves under boundary conditions (25) for the considered unit cell sizes.

Results are reported in figures 9 and 10. The values of $\bar{U}$ and $\left\langle U^{r}\right\rangle$ are normalized by $U_{0}$, the average strain energy of the inclusion material under an applied tension of $800 \mathrm{MPa}$. With both sets of boundary conditions the average macroscopic elastic energy $\bar{U}$ at convergence is already achieved for a cell size $\xi=8.43$, this can be explained by the fact that with mixed boundary conditions (24) the convergence of the effective elastic properties is faster in the case of uniform static or kinematic boundary conditions [26]. In the case of boundary conditions (24) the average elastic energy due to residual stresses $\left\langle U^{r}\right\rangle$ decreases significantly (about 25\%) with the cell size for $5.69 \leq \xi \leq 11.29$, while its value varies slightly (about $6 \%$ ) between $\xi=11.29$ and $\xi=14.12$ (figure 9). With boundary conditions (25), $\left\langle U^{r}\right\rangle$ exhibts an opposite trend: it increases with the cell size with variations of about $75 \%$ for $5.69 \leq \xi \leq 11.29$ and of only $1 \%$ between $\xi=11.29$ and $\xi=14.12$. The convergence of $\frac{\left\langle U^{r}\right\rangle}{U_{0}}$ implies that the unit cell with $\xi=14.12$ can be considered a RVE for the elastoplastic uniaxial behaviour up to the considered applied average stress or strain.

The steadiness of the elastic energy due to residual stresses with respect to the cell size proves to be a valid criterion to evaluate the RVE for the uniaxial elastoplastic behaviour. This assessment is confirmed by the fact that the stress-strain curves obtained under different boundary conditions, (24) and (25), almost coincide up to $\langle\epsilon\rangle_{11}=0.05$, as shown in figure 7 as well.

The cubic symmetry of the composite was verified for the elastoplastic behav- 


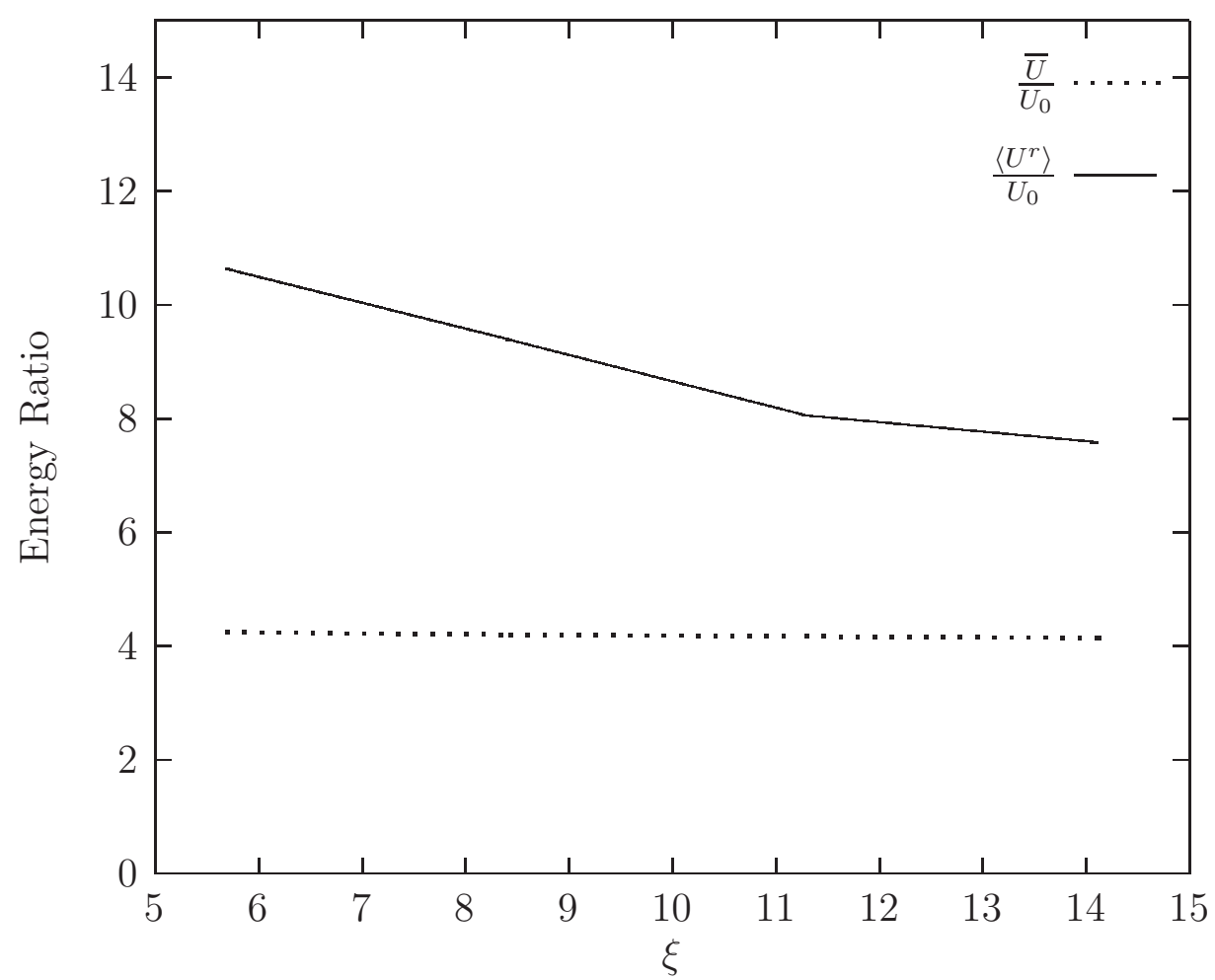

Fig. 9. Normalized average macroscopic elastic energy $\frac{\bar{U}}{U_{0}}$ and normalized average elastic energy due to residual stresses $\frac{\left\langle U^{r}\right\rangle}{U_{0}}$ as functions of the unit cell size under boundary conditions (24) with an applied tensile stress of $800 \mathrm{MPa}$.

iour as well. Pure traction simulations were carried out for the 1, 2, 3 directions and no significant difference could be observed in the effective stress-strain curves (boundary conditions of the type (25) were applied as they are less demanding in terms of computing time since they do not include the face planarity condition). The uniaxial tensile behaviour was determined for all the considered compositions (table 2 ) on unit cells with $\xi \approx 14$. The strengthening effect of the particles on the effective stress-strain curves (up to $\langle\epsilon\rangle_{11}=0.05$ ) can be observed in figure 11 .

\section{Application to experimental data}

The model was applied to the real case of the 2124 aluminum alloy reinforced with $\mathrm{SiC}$ particles studied in [27]. The composite contains 17 vol.\% SiC particles of an average size of $1.4 \mu \mathrm{m}$. The authors carried out traction tests at different temperatures both on the matrix metal and on the composite. The negligible amount of reaction products between 2124 aluminum alloy and $\mathrm{SiC}$ particles [28] allows to assume for the matrix material in the composite the uniaxial stress-strain curve obtained by testing the bulk material. 


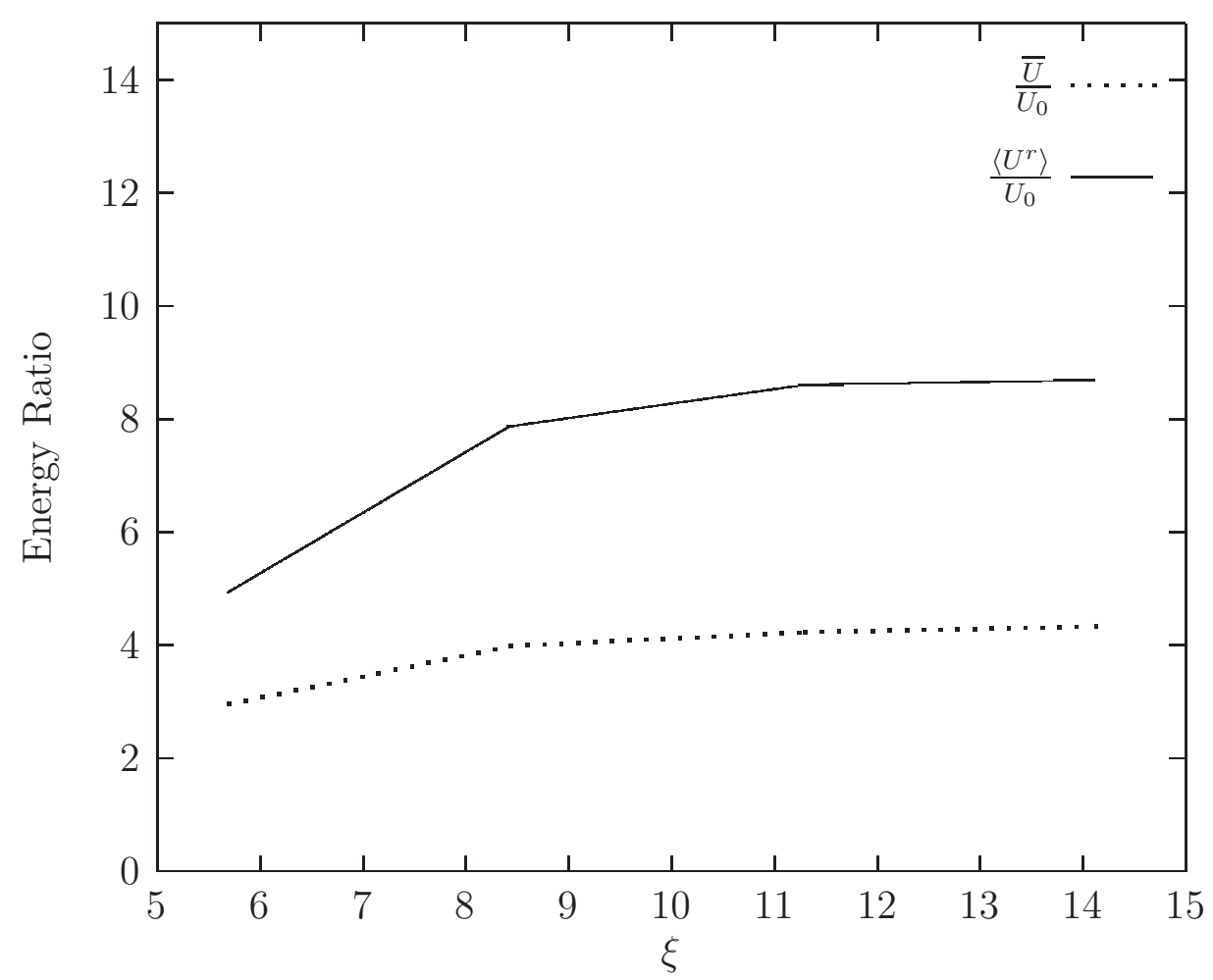

Fig. 10. Normalized average macroscopic elastic energy $\frac{\bar{U}}{U_{0}}$ and normalized average elastic energy due to residual stresses $\frac{\left\langle U^{r}\right\rangle}{U_{0}}$ as functions of the unit cell size under boundary conditions (25) with an applied tensile strain of 0.05 .

For the purposes of this work the mechanical behaviour at room temperature is considered and tensile tests are simulated under boundary conditions (25) up to $5 \%$ strain, a value which is compatible both with the small displacement assumption and with the chosen mechanical behavior. Moreover in this strain interval it is reasonable to assume that damage can be neglected thus the model hypotheses of particle and matrix integrity and of perfect interfaces between the two are acceptable.

The homogenization model consisted of a unit cell of $3375 \mu \mathrm{m}^{3}$ (corresponding to an edge length of $15 \mu \mathrm{m}$ ) containing about 380 particles. The particle average volume was $1.5 \mu \mathrm{m}^{3}$ with $0.1 \mu \mathrm{m}^{3}$ standard deviation, corresponding to an average equivalent diameter of $1.42 \mu \mathrm{m}$. The $\mathrm{SiC}$ particles were assigned linear elastic behaviour; the values $E=415 \mathrm{GPa}$ and $\nu=0.16$ were chosen [29]. The matrix material was assigned an elastoplastic behaviour following the Von Mises criterion with isotropic hardening (13). The strain hardening profile was extracted from the experimental uniaxial stress-strain curves at room temperature reported by the authors.

The stress-strain curve obtained by homogenization is compared with the experimental one in figure 12, it can be noted that there is full agreement between the two up to $\langle\epsilon\rangle_{11}=0.05$. 


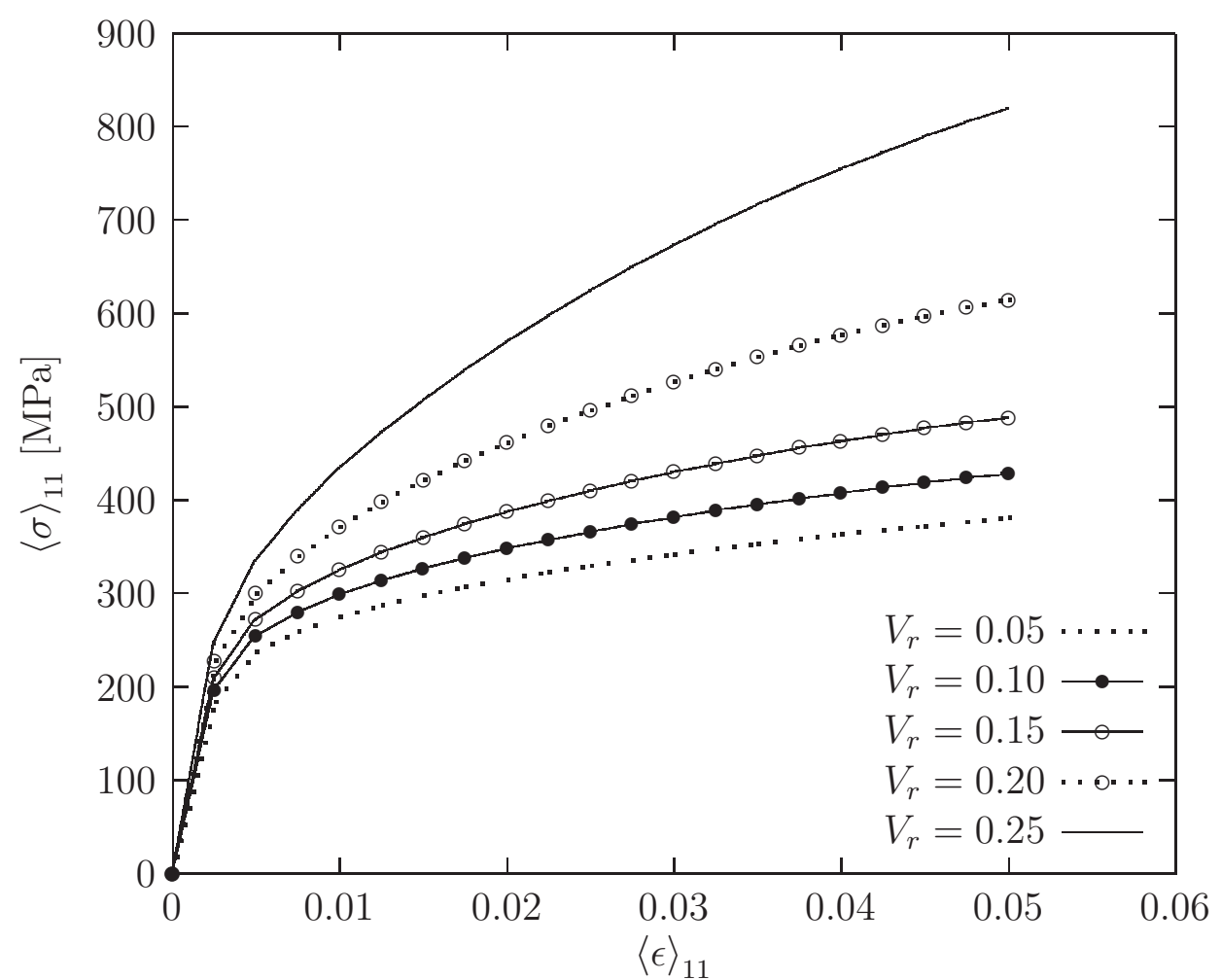

Fig. 11. Effective uniaxial stress-strain curves of the different composites.

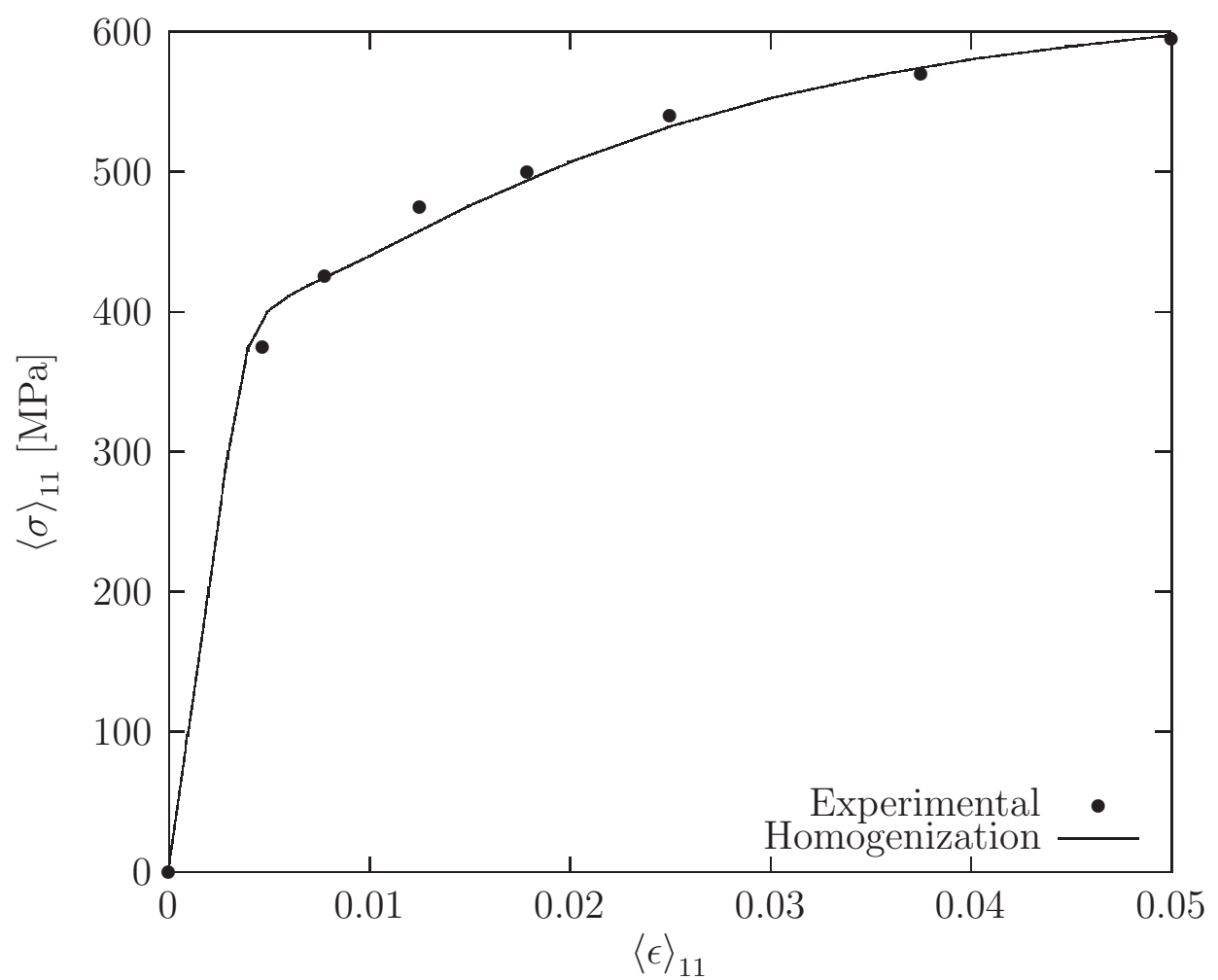

Fig. 12. Comparison between the Al2124-17 vol.\% SiC experimental stress-strain curve and that obtained by homogenization. 


\section{Summary and conclusions}

A novel technique to generate three dimensional microstructural models for the homogenization of the mechanical properties of particle reinforced composites was developed. Particles are created in an already existent tetrahedral mesh, obtained by means of the CDT. Thus models for materials with different compositions can be developed from the same mesh without the need to draw and mesh the model geometry for each of the considered reinforcement volume fractions. Moreover a polyhedral reinforcement morphology is obtained, with particles resembling those of the ceramic powders often used as reinforcement.

Homogenization was performed for the thermoelastic and the elastoplastic behaviour of a typical PRMMC. The obtained RVE size for the elastic behaviour is consistent with analogous estimations reported in previous works and both the effective elastic properties and the CTE comply with the respective bounds. To evaluate the elastoplastic behaviour in simple traction, simulations were carried out by applying different boundary conditions. The RVE size at the imposed stress (strain) level was assessed by studying the dependence of the average elastic energy related to residual stresses on the unit cell size. The RVE was estimated for $\langle\sigma\rangle_{i i}=800 \mathrm{MPa}$, corresponding to $\langle\epsilon\rangle_{i i} \approx 0.05$ and, as expected, was significantly larger than that for elastic behaviour, containing about 700 particles.

Lastly the developed model was applied to a PRMMC whose elastoplastic properties are reported in literature. The effective stress-strain curve obtained by homogenization fits very well the experimental data, proving the consistency of the proposed approach. Nevertheless, the present model does not account for particle size and interparticle distribution, which may affect the stress-strain curve [30, 31]. To examine the limits of the model, additional experimental data with different reinforcement volume fractions and characteristic particle sizes need to be considered.

Some further developments of this technique can be foreseen. The algorithm to generate particles can be improved to meet wider requirements in terms of reinforcement volume fraction and of particle size distribution. To generate particles having aspect ratios larger than one, sets of two or more vertices, instead of single vertices, could be chosen as nucleation sites while larger particles could be generated by assigning the inclusion material not only to the elements which share the nucleation point, or points, but also to the surrounding layers of tetrahedra. Both solutions could then be extended to study materials in which particles are not randomly oriented. Moreover other material constitutive behaviours could be taken into account (e.g. elastoplasticity of the particles or viscoplasticity of the matrix) along with the effect of the residual stresses deriving from the fabrication process. 


\section{Acknowledgements}

The authors thank Dr. J. Cugnoni and Dr. F. Colpo for the useful discussion. The work was sponsored by EMPA and EPFL.

\section{References}

[1] H. J. Böhm, A Short Introduction to Basic Aspects of Continuum Mechanics, Tech. rep., Institute of Lightweight Structures and Aerospace Engineering, Vienna University of Technology, Austria (1998).

[2] H. D. Espinosa, P. D. Zavattieri, A grain level model for the study of failure initiation and evolution in polycrystalline brittle materials. Part I: Theory and numerical implementation, Mechanics of Materials 35 (3-6) (2003) 333-364.

[3] N. Chawla, X. Deng, D. Schnell, Thermal expansion anisotropy in extruded $\mathrm{SiC}$ particle reinforced 2080 aluminum alloy matrix composites, Materials Science and Engineering: A 426 (1-2) (2006) 314-322.

[4] A. Borbely, H. Biermann, O. Hartmann, FE investigation of the effect of particle distribution on the uniaxial stress-strain behaviour of particulate reinforced metal-matrix composites, Materials Science and Engineering A 313 (1-2) (2001) 34-45.

[5] T. Iung, M. Grange, Mechanical behaviour of two-phase materials investigated by the finite element method: necessity of three-dimensional modeling, Materials Science and Engineering A 201 (1-2) (1995) L8-L11.

[6] H. J. Böhm, W. Han, Comparisons between three-dimensional and twodimensional multi-particle unit cell models for particle reinforced metal matrix composites, Modelling and Simulation in Materials Science and Engineering 9 (2001) 47-65.

[7] P. Kenesei, A. Borbely, H. Biermann, Microstructure based threedimensional finite element modeling of particulate reinforced metalmatrix composites, Materials Science and Engineering A 387-389 (2004) 852-856.

[8] A. Borbely, F. F. Csikor, S. Zabler, P. Cloetens, H. Biermann, Threedimensional characterization of the microstructure of a metal-matrix composite by holotomography, Materials Science and Engineering A 367 (1-2) (2004) 40-50.

[9] N. Chawla, R. Sidhu, V. Ganesh, Three-dimensional visualization and microstructure-based modeling of deformation in particle-reinforced composites, Acta Materialia 54 (6) (2006) 1541-1548.

[10] R. Sidhu, N. Chawla, Three-dimensional (3D) visualization and microstructure-based modeling of deformation in a Sn-rich solder, Scripta Materialia 54 (9) (2006) 1627-1631. 
[11] C. Gonzalez, J. Segurado, J. LLorca, Numerical simulation of elastoplastic deformation of composites: evolution of stress microfields and implications for homogenization models, Journal of the Mechanics and Physics of Solids 52 (7) (2004) 1573-1593.

[12] H. Si, TetGen A Quality Tetrahedral Mesh Generator and ThreeDimensional Dalaunay Triangulator, Version 1.3, User's Manual (2005).

[13] E. L. Hinrichsen, J. Feder, T. Jssang, Geometry of random sequential adsorption, Journal of Statistical Physics 44 (5 - 6) (1986) 793-827.

[14] M. D. Rintoul, S. Torquato, Reconstruction of the Structure of Dispersions, Journal of Colloid and Interface Science 186 (2) (1997) 467-476.

[15] J. Feder, Random sequential adsorption, Journal of Theoretical Biology 87 (2) (1980) 237-254.

[16] J. D. Sherwood, Packing of spheroids in three-dimensional space by random sequential addition, Journal of Physics A: Mathematical and General 30 (24) (1997) L839-L843.

[17] R. Hill, Elastic properties of reinforced solids: Some theoretical principles, Journal of the Mechanics and Physics of Solids 11 (5) (1963) 357-372.

[18] H. T. Liu, L. Z. Sun, Effects of thermal residual stresses on effective elastoplastic behavior of metal matrix composites, International Journal of Solids and Structures 41 (8) (2004) 2189-2203.

[19] Z. Hashin, S. Shtrikman, A variational approach to the theory of the elastic behaviour of multiphase materials, Journal of the Mechanics and Physics of Solids 11 (2) (1963) 127-140.

[20] V. Levin, Thermal Expansion Coefficients of Heterogeneous Materials, Mekhanika Tverdogo Tela 2 (1967) 88-94.

[21] R. Schapery, Thermal Expansion Coefficients of Composite Materials Based on Energy Principles, Journal of Composite Materials 2 (1968) 380-404.

[22] W. J. Drugan, J. R. Willis, A micromechanics-based nonlocal constitutive equation and estimates of representative volume element size for elastic composites, Journal of the Mechanics and Physics of Solids 44 (4) (1996) 497-524.

[23] P. M. Suquet, Elements of Homogenization for Inelastic Solid Mechanics, Lecture Notes in Physics 272 (1985) 194-278.

[24] T. Kanit, S. Forest, I. Galliet, V. Mounoury, D. Jeulin, Determination of the size of the representative volume element for random composites: statistical and numerical approach, International Journal of Solids and Structures 40 (13-14) (2003) 3647-3679.

[25] C. C. Swan, Techniques for stress- and strain-controlled homogenization of inelastic periodic composites, Computer Methods in Applied Mechanics and Engineering 117 (3-4) (1994) 249-267.

[26] S. Hazanov, C. Huet, Order relationships for boundary conditions effect in heterogeneous bodies smaller than the representative volume, Journal of the Mechanics and Physics of Solids 42 (12) (1994) 1995-2011.

[27] E. Martin, A. Forn, R. Nogue, Strain hardening behaviour and tempera- 
ture effect on Al-2124/SiCp, Journal of Materials Processing Technology 143-144 (2003) 1-4.

[28] C. Badini, G. M. La Vecchia, P. Fino, T. Valente, Forging of 2124/SiCp composite: preliminary studies of the effects on microstructure and strength, Journal of Materials Processing Technology 116 (2-3) (2001) 289-297.

[29] R. G. Munro, Material Properties of a Sintered alpha-SiC, Journal of Physical and Chemical Reference Data 26 (1997) 1195-1203.

[30] M. Kouzeli, A. Mortensen, Size dependent strengthening in particle reinforced aluminium, Acta Materialia 50 (1) (2002) 39-51.

[31] Z. Ling, L. Luo, B. Dodd, Experimental study on the formation of shear bands and effect of microstructure in $\mathrm{Al}-2124 / \mathrm{SiCp}$ composites under dynamic compression, Journal de Physique IV C8 (1994) 453-457. 